\title{
Resonator coupled Josephson junctions; parametric excitations and mutual locking
}

\author{
Jensen, H. Dalsgaard; Larsen, A.; Mygind, Jesper
}

Published in:

I E E E Transactions on Magnetics

Link to article, DOI:

$10.1109 / 20.133931$

Publication date:

1991

Document Version

Publisher's PDF, also known as Version of record

Link back to DTU Orbit

Citation (APA):

Jensen, H. D., Larsen, A., \& Mygind, J. (1991). Resonator coupled Josephson junctions; parametric excitations and mutual locking. I E E E Transactions on Magnetics, 27(2, 4), 3355-3358. https://doi.org/10.1109/20.133931

\section{General rights}

Copyright and moral rights for the publications made accessible in the public portal are retained by the authors and/or other copyright owners and it is a condition of accessing publications that users recognise and abide by the legal requirements associated with these rights.

- Users may download and print one copy of any publication from the public portal for the purpose of private study or research.

- You may not further distribute the material or use it for any profit-making activity or commercial gain

- You may freely distribute the URL identifying the publication in the public portal

If you believe that this document breaches copyright please contact us providing details, and we will remove access to the work immediately and investigate your claim. 
RESONATOR COUPLED JOSEPHSON JUNCTIONS;

PARAMETRIC EXCITATIONS AND MUTUAL LOCKING

\author{
H. Dalsgaard Jensen \\ Danish Institute of Fundamental Metrology, DK-2800 Lyngby, Denmark \\ A. Larsen ${ }^{\mathbf{k}}$
}

Physikalisch-Technische Bundesanstalt, D-3300 Braunschweig, Federal Republic of Germany

J. Mygind

Physics Laboratory I, B 309, Technical University of Denmark, DK-2800 Lyngby, Denmark

\begin{abstract}
Self-pumped parametric excitations and mutual locking in systems of Josephson tunnel junctions coupled to multi-mode resonators are reported. For the very large values of the coupling parameter, obtained with small $\mathrm{Nb}-\mathrm{Al}_{2} \mathrm{O}_{3}-\mathrm{Nb}$ junctions integrated in superconducting microstrip resonators, the $\mathrm{dc}$ $I V$-characteristic shows an equidistant series of current-steps generated by subharmonic pumping of the fundamental resonator mode. This is confirmed by measurements of frequency and linewidth of the emitted Josephson radiation.
\end{abstract}

\section{Introduction}

The Josephson junction is a highly nonlinear element and its interaction with a single resonance circuitry have been studied both theoretically and experimentally; see Ref. 1 and references therein. Here we report a study of self-induced steps observed in the dc $I V$-characteristic of a small tunnel junction (i.e. with spatial dimensions shorter than $\lambda_{J}$, the Josephson penetration depth) strongly coupled to an open-ended section of a low-loss superconducting microstrip line. This multi-mode (nearly full-wave) resonant system allows us to effectively tune out the inherent junction capacitance and thus to achieve exceptionally large values of the coupling parameter.

For a given mode the coupling parameter, which also depends on the critical current of the junction and the total system loss, can be varied by applying an external magnetic field to the junction, or by changing the system temperature. With strong coupling we observe a series of large self-induced steps (PMS, parametrically generated mode steps) excited by parametric subharmonic pumping of the fundamental resonant mode. In narrow parameter ranges also half-integer PMS are stable. The self-induced mode steps (MS), known from Josephson junctions interacting with resonant transmission line modes in the weak coupling limit ${ }^{2-5}$, are also observed. Our measurements show that in the multi-mode system with stronger coupling and large harmonic overlap between the mode resonances the MS seem to be dominated by the PMS.

Parametrically generated steps in a single resonance system have been observed in a resistively shunted SQUID ${ }^{6}$.

The junction-resonator system is ideally suited for the basic study of nonlinear dynamics. Unlike a bare junction the system is of third or higher order, so bifurcations and chaos are likely to occur ${ }^{7}$. Stability analysis of single as well as arrays of strongly coupled systems is of importance especially for integration of high current density submicron junctions in millimeter wave circuits where spurious resonances in the embedding network are very likely. (a)

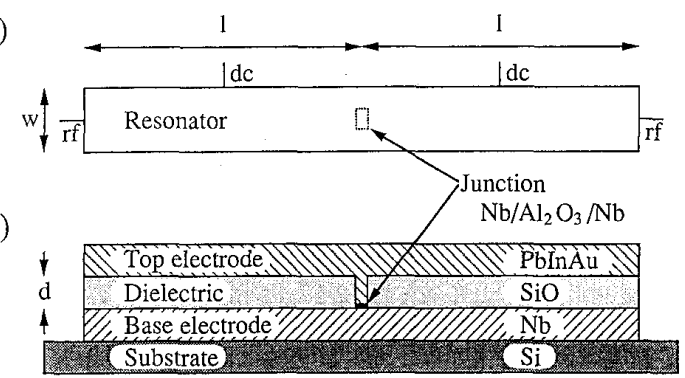

(c)

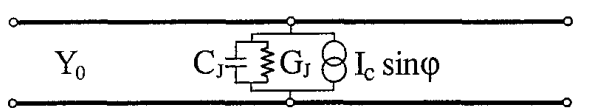

Figure 1. (a) Top view, (b) side view, and (c) transmission line model of a microstrip resonator with a small Josephson tunnel junction. The width, half length and thickness of dielectric of the microstrip are $w, \ell$, and $d$, respectively. The dimensions are out of scale.

\section{Experimental System}

The junction fabrication and experimental setup has been described in detail elsewhere ${ }^{8}$. Fig. 1 shows the experimental sample, an all-niobium $\left(\mathrm{Nb} / \mathrm{Al}_{2} \mathrm{O}_{3} / \mathrm{Nb}\right)$ sandwich Josephson junction integrated in the center of a superconducting microstrip resonator. The base Nb-layer of the junction is extended to form the bottom electrode of the resonator. The top electrode is formed by a third lead-indium-gold ( $\mathrm{PbInAu}$ ) alloy film, which is connected to the upper Nb-layer of the junction through a window in the SiO-layer. The SiO-layer is the separating dielectric in the microstrip resonator.

Eight $25.4 \mathrm{~mm} \times 12.7 \mathrm{~mm}$ samples are made in each batch on a 3-inch diameter high resistivity silicon wafer. In a given batch the junctions show less than $5 \%$ variation in the critical current $I_{c}$. Only the resonator dimensions differ between the samples on a wafer, using $\ell=2.75,4.0$, and $5.5 \mathrm{~mm}$, and $w$ $=100$ and $200 \mu \mathrm{m}$. The size of the rectangular junction is $20 \mu \mathrm{m}$ $\times 30 \mu \mathrm{m}$. With $\ell=5.5 \mathrm{~mm}$ the resonance frequency is about 8 GHz. On the samples used in our study of mutual phaselocking of Josephson tunnel junctions two identical resonators each containing one junction are placed 4 or $8 \mu \mathrm{m}$ from each other. The separation, however, is made much larger than $d$ in order to achieve a small mutual coupling between the resonators. 
In the experimental setup all sensitive electronic parts are powered from batteries and large efforts have been made to reduce interference from external noise sources. The wires leading to the junction are carefully filtered, twisted pairs. The importance of the stability of the three dc bias parameters (temperature $T$, applied magnetic field $B$; and bias current $I$ ) is illustrated by the measured (typical) frequency tuning rates of the Josephson oscillation when biased on one of the PMS: $\Delta f / \Delta T=-0.15 \mathrm{MHz} / \mathrm{mK}, \Delta f / \Delta B=0.5 \mathrm{MHz} / \mu \mathrm{T}$, and $\Delta f / \Delta I=2 \mathrm{MHz} / \mu \mathrm{A}$ (with a dynamic resistance of about 10 $\mathrm{m} \Omega$ ), respectively.

In order to characterize the samples and as a diagnostic tool for eliminating noise sources we measure the escape rate from the zero-voltage state. Usually we collect 50.000 escape events in an automated data processing system. Typically we find an effective temperature a few tenths of a Kelvin above the bath temperature. The escape rate measurements allows us to correct for noise suppression of the critical current.

The microwave radiation emitted from the steps had a very narrow linewidth, down to a few $\mathrm{kHz}$, so a precise measurement of the Josephson frequency and hence the step voltage is possible. For junction $\mathrm{H} 5-2 \mathrm{~A}$ the frequency at MS1 is $\sim 8.4 \mathrm{GHz}$ corresponding to a voltage of $17.4 \mu \mathrm{V}$, see Fig. 2 . Radiation at the same frequency has been measured on all stable steps and the variation in frequency from step to step is much smaller than the variation of the mode resonances $f_{n} \cdot{ }^{8}$, thus all the steps are harmonic replica of the fundamental mode step, MS1, a strong evidence for our interpretation of the harmonic steps being generated by parametric subharmonic pumping of the fundamental mode step. We use the abbreviation PMS $k$ for these steps. Label $k$ indicates that it is the $k$ th subharmonic of the Josephson oscillation which pumps the fundamental mode resonance.

\section{Single Resonance Theory}

Self-induced steps may be regarded as a sort of regenerative rf-induced steps. At resonance the field in the resonator is large, and the junction receives the resonator signal as driven from a microwave generator, which in turn is powered by the junction itself. This argument was used to explain the form and size of self-induced steps ${ }^{1}$ in the much simpler system of a small Josephson junction coupled to a circuit with only a single resonance. The important assumption is that harmonics of the Josephson oscillations are negligible when operating near resonance. For use in the following we shall refer to these steps as single resonator-induced steps ( $\mathrm{RS}$, for brevity).

In the single resonance theory the coupling parameter $z$, which relates the coupling strength to both junction and resonator properties, is defined as

$$
z=\frac{I_{c}}{V_{r} \operatorname{Re} Y_{\Sigma}}
$$

where $I_{c}$ is the critical current of the junction, and $V_{T}=(h / 2 e) f_{r}$ is the dc voltage corresponding to the resonance frequency $f_{r}$ and Re $Y_{\Sigma}$ the real part of the total admittance of the resonator including the passive admittance of the junction.

The single resonance theory also predicts parametric subharmonic excitation of the single resonator system ${ }^{1}$. When the junction is (current) biased, say, on the quasi-particle curve

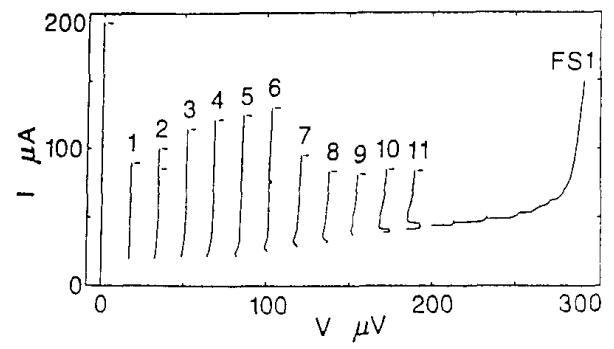

Figure 2. Typical recorded dc $I V$-characteristic (sample H5-2A, $\ell=5.5 \mathrm{~mm}, w=200 \mu \mathrm{m}$ ) showing the first mode step, MS1 and a series of parametrically generated mode steps, PMS $k$. The temperature was $4.2 \mathrm{~K}$ and an external magnetic field, $B=272$ $\mu \mathrm{T}$ was applied. The arrows indicate the current at which the junction switches from the step. The structure to the right is the first Fiske step at $295 \mu \mathrm{V}$. The position of the foot-like structures visible on higher numbered PMS $k$ agree with the voltages of the corresponding mode steps, $\mathrm{MS} n$.

at a dc voltage equal to $n V_{r}$, the resonator may be parametrically excited. This results in parametrically induced resonator steps (PRS) at these voltages, too. However, the analysis (Figures 12.6, for $m=2$, and 12.10 in Ref. 1) shows that only the second PRS is soft excited, i.e. it will appear without external interference. The other PRS are hard excited, and will appear only, if an oscillation with the particular frequency already exists with some minimum amplitude. As mentioned such so-called harmonic steps have already been observed ${ }^{6}$.

\section{Multi-Mode System}

Fig. 1 includes a microwave model of the experimental system. The resonator is an open-ended transmission line with characteristic admittance $Y_{0}$ and propagation constant $\gamma=\alpha+j \beta$. The junction is modelled by a parallel connection of the active element, the supercurrent channel $I_{S}=I_{c} \sin \varphi$ and the passive admittance $Y_{J}(f)=G_{J}+j 2 \pi f C_{J}$, representing the junction capacitance $C_{J}$ and the (in general nonlinear) quasi-particle conductance $G_{J}$. When dc-biased at a voltage $V$ the corresponding Josephson oscillation has the fundamental frequency $f=(2 e / h) V$ where $-e$ is the electron charge and $h$ is Plancks constant. Here we neglect the admittance, $Y_{S}$, of the bare Josephson element which seems to play a minor role ${ }^{8}$. element is

The total admittance $Y_{\Sigma}$ seen by the bare Josephson

$$
Y_{\Sigma}=Y_{J}+2 Y_{0} \tanh \gamma \ell
$$

and resonance occurs when the imaginary part, $\operatorname{Im} Y_{\Sigma}$, equals zero.

The resonance condition (low-loss limit) can be written as

$$
\tan \pi \frac{f}{f_{e}}=-\pi \frac{C_{J}}{C_{e}} \frac{f}{f_{e}}
$$

where $C_{e}=\epsilon_{r} \epsilon_{o}(2 \ell w) / d$ is the total capacitance of the microstrip resonator, and $\epsilon_{\circ}$ is the vacuum permittivity. The resonance frequency of the unloaded resonator (without the junction), when one full wavelength of the oscillation equals the length of the resonator. $f_{e}$ is given by

$$
f_{e}=\frac{c_{0}}{2 \ell \sqrt{\epsilon_{\tau} \chi_{r}}}
$$


where $c_{0}$ is the light velocity in vacuum, $\epsilon_{r}$ the relative dielectric constant of the insulator, and $\chi_{r}=\left(d+\lambda_{a}+\lambda_{b}\right) / d$ accounts for the penetration of magnetic fields in the resonator electrodes. With the resonator dimensions used here, the characteristic admittance, $Y_{0}$, is typically a few $\Omega^{-1}$.

The solutions to this equation are called $f_{n}$; the resonance frequency of the $n$th mode of the system. In the weak coupling limit the corresponding current singularities in the dc $I V$-characteristic will be the $n$th mode step (MSn).

In analogy with the single resonance theory the coupling parameter for the $n$th mode may be given as

$$
z_{n}=\frac{I_{c}}{V_{n}\left(G_{J}+G_{e}\right)}
$$

in the low-loss limit. Here $V_{n}=(h / 2 e) f_{n}$ is the dc voltage corresponding to the mode frequency $f_{n}$.

The quality factor $Q_{n}$ of the $n$th mode is

$$
Q_{n}=\pi \frac{f_{n}}{f_{e}} \frac{f_{e} C_{J}+G_{e} /(\alpha \ell)}{G_{J}+G_{e}}
$$

The microstrip losses in $G_{e}$ are all contained in the real part $\alpha$ of the propagation constant, where $\alpha$ is the sum of three terms: surface losses $\alpha_{c}$, dielectric losses $\alpha_{d}$, and radiation losses $\alpha_{r}{ }^{8}$.

In the experiment we have chosen a long and wide resonator. Here the first harmonics $n f_{1}$ of the fundamental resonance are closer to the $n$th mode frequency $f_{n}$ than the half-power bandwidth of the mode resonances. The resonator, loaded by the junction passive admittance, therefore comprises many high- $Q$ resonant modes being spaced sufficiently constant in frequency to allow for a considerable overlap of harmonics.

Fig. 2 shows a typical low voltage part of in the dc $I V$-characteristic (junction H5-2A). Similar characteristics have been obtained for all the other junctions on the wafer. Generally all junctions on a given wafer had the same parameters, e.g. area $30 \times 20 \mu \mathrm{m}^{2}$ and $I_{c}=775 \mu \mathrm{A}$ at $4.2 \mathrm{~K}$. The critical current is corrected $(\sim 3 \%)$ for noise suppression found from escape rate measurements as described above. The junctions showed nearly perfect magnetic interference pattern indicating an undisturbed tunneling barrier with constant current density. From the $I_{c}(B)$ minima and from the position of the Fiske step, at $295 \mu \mathrm{V}$, we derive the magnetic thickness, $d_{J}=225 \mathrm{~nm}$, the capacitance $C_{J}$ $=28 \mathrm{pF}$, and the Josephson penetration depth, $\lambda_{J}=30 \mu \mathrm{m}$ at $4.2 \mathrm{~K}$. The normalized length $L / \lambda_{J}=1$ and the maximum plasma frequency $f_{p}=46 \mathrm{GHz}$.

\section{Spectral Linewidth}

The full half-power linewidth $\Delta \nu$ of the Josephson oscillations in a bias point $\left(I_{\mathrm{dc}}, V_{\mathrm{dc}}\right)$ is given by

$$
\Delta \nu=4 \pi k_{B} T\left(\frac{2 e}{h}\right)^{2} \frac{R_{d}^{2}}{R_{\mathrm{dc}}}
$$

where $R_{d}$ is the differential resistance, $R_{\mathrm{dc}}=V_{\mathrm{dc}} I_{\mathrm{dc}}^{-1}$ is the dc resistance, and the $k_{B}$ is Boltzmann's constant. The frequency and the linewidth $\Delta \nu$ of the emitted radiation with the junction biased on a step can be recorded on the spectrum analyzer. In Fig. 3 is shown $\Delta \nu$ in $\mathrm{kHz}$ versus $R_{d}^{2} / R_{\mathrm{dc}}$ in $\mathrm{m} \Omega$ for sample H5-2A biased on different points along MS1. At large values of

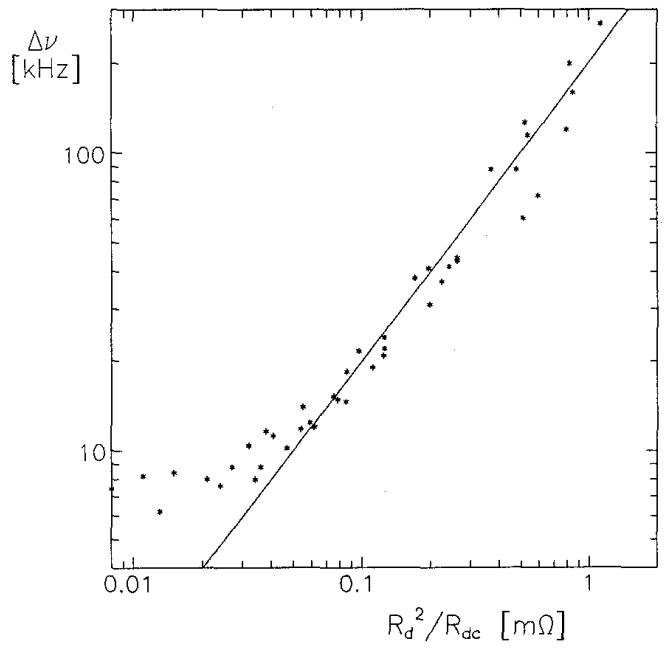

Figure 3. Half-power linewidth $\Delta \nu$ versus the differential resistance $R_{d}$ squared divided by the dc resistance $R_{d c}$ (see text) of the radiation emitted from sample $\mathrm{H} 5-2 \mathrm{~A}$ with the junction biased on MS1. The inserted line has unity slope. The experimental conditions are similar to Fig. 2 .

$\Delta \nu$ the points group around a straight line with unity slope as predicted by Eq. (7). A resolution bandwidth of $10 \mathrm{kHz}$ on the spectrum analyzer was used. This explains the saturation seen in the figure. From the measured $\Delta \nu, R_{d}$, and $R_{\mathrm{dc}}$ the noise temperature $T$ in Eq. 7 can be found to $4.5 \pm 1.5 \mathrm{~K}$ in agreement with the bath temperature $(4.2 \mathrm{~K})$ indicating a low level of external noise.

\section{Mutual Locking}

Here we use samples with two resonator/junction systems, both exhibiting the step structures described above. One will be denoted A, the second B in the following. Biasing junction A on MS1, and tracing out MS1 for junciton B, frequency pulling and phaselocking is observed.

Fig. 4 shows a current-voltage trace of MS1 for junction $B$ when junction $A$ is biased at different points also on MS1. The leftmost trace shows MS1 with junction A biased at zero current/zero voltage. In the next trace junction $A$ is biased at the point on MS1 where it radiates a signal with frequency 8.200 $\mathrm{GHz}$ with junction $\mathrm{B}$ biased at zero current/zero voltage. The circle on the trace denotes the point at which the phaselocked signal has the frequency $8.200 \mathrm{GHz}$. For the successive traces junction $\mathrm{A}$ is biased at the point where it radiates at 8.220 , $8.240, \ldots, 8.380 \mathrm{GHz}$, respectively.

The maximum range of locking $(\Delta I=40 \mu \mathrm{A})$ is limited by the height of the step, and are centered around the point at which the locked signal frequency equals that of junction $\mathrm{A}$ unlocked (circles in Fig. 4). Locking occurs when the frequencies of the signals from the two junctions differ less than $120 \mathrm{MHz}$ at low bias to $70 \mathrm{MHz}$ at high bias, and frequency pulling is clearly observed on the spectrum analyzer display already at a frequency difference of about $200 \mathrm{MHz}$.

Fig. 5 shows spectra of the locking process, when the bias current in junction B is increased. As seen from the figure, the power of the phaselocked signal is larger than the sum of the two signals before locking. Also linewidth narrowing has been 


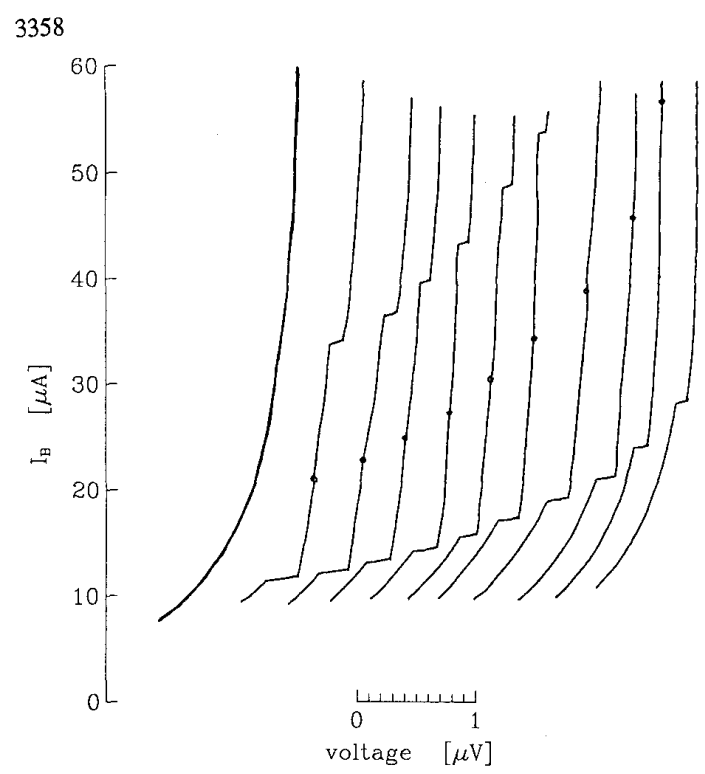

Figure 4. Current-voltage trace of the first mode step MS1 for junction $\mathrm{H} 5-2 \mathrm{~B}$, with junction $\mathrm{H} 5-2 \mathrm{~A}$ biased at different points along its MS1. The leftmost trace is for junction $A$ biased at zero current/zero voltage, in the successive traces junction $A$ is biased at the point where it radiated a signal with frequency $8.200,8.220, \ldots, 8.380$, when junction $B$ is biased at zero current/zero voltage. Circles denote the point where the locked signal frequency equals that of junction $\mathrm{A}$ unlocked.

observed, however, it has been difficult to characterize due to excessive bias current noise from one of the dc current sources used.

The maximum locking range observed corresponds to about $20 \%$ of $I_{c}$, however, with the supercurrent suppressed by a magnetic field. Locking is also observed when biasing the two junctions on other combinations of steps. First of all, interchanging the two junctions, keeping junction $\mathrm{B}$ at fixed bias and tracing MS1 for junction A, gives virtually no difference in locking ranges. The same is the case for the combinations of junction A and B on the steps MS1 and MS(-1). Also when biasing junction $A$ on a higher order step, frequency pulling and locking is observed when tracing out a step for junction B. Generally, whenever the frequency difference of the signal radiated from the two states differ less than the order $100 \mathrm{MHz}$, locking occurs. Frequency pulling and phaselocking was also observed when biasing junction $A$ on the step believed to be MS2, where no signal at $8 \mathrm{GHz}$ is radiated, and tracing out MS1 for junction $B$. This is in agreement with the measurements of a large harmonic content of the Josephson oscillation on the steps. With both junctions biased on the supposed MS2, a maximum locking range of the same order as for MS1 is found.

\section{Acknowledgements}

We thank N. Grønbech-Jensen, R. Fromknecht, J. Niemeyer, R. Pöpel, and M.R. Samuelsen for stimulating discussions, and Anders Grunnet-Jepsen and Axel Morgenstjerne for assistance with some of the measurements. This work was partly funded by the Commission of the European Communities, the Danish Research Academy, and the Danish National Council of Metrology.

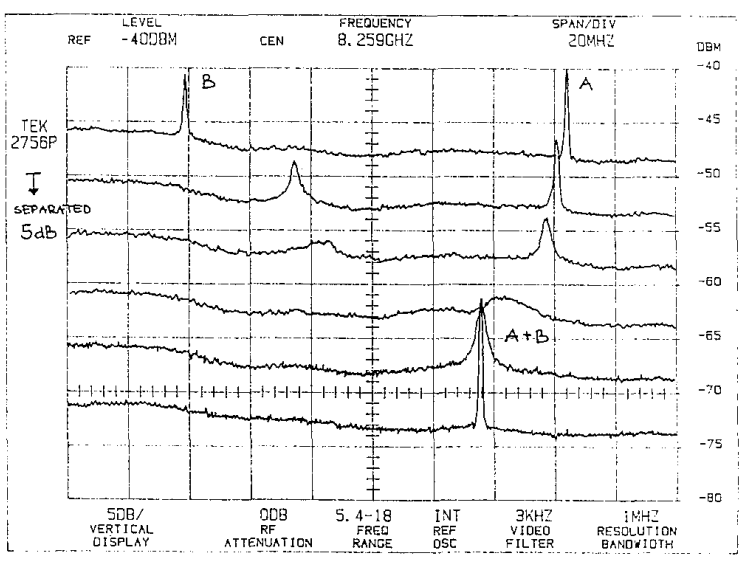

Figure 5. Spectra of the locking process. The bias current is changed for junction $B$, pulling the signal frequency of junction A. After locking the power of the locked signal is larger than the sum of the two unlocked signals.

\section{References}

*. Present address: Telecommunication Research Laboratory, DK-2970 Hørsholm, Denmark

1. K. K. Likharev, Dynamics of Josephson Junctions and Circuits, Chapter 12, Gordon and Breach, New York, 1986.

2. G. Paterno, A. M. Cucolo, and G. Modestino, "Resonant modes for a Josephson junction coupled to a stripline resonator", pp. 215-216 in LT-17, U. Eckern, A. Schmid, W. Weber, and H. Wühl, Eds., North-Holland, Amsterdam, 1984.

3. A. D. Smith, B. J. Dalrymple, A. H. Silver, R. W. Simon, and J. F. Burch, "Microstrip resonances in superconducting circuits", IEEE Trans. Magn., MAG-23, 796-799, 1987.

4. A. Raisanen, W. McGrath, P. Richards, and F. Lloyd, "Broad-band rf match to a millimeter-wave SIS quasi-particle mixer", IEEE Trans. Microwave Theory Techn., MTT-33, $1495-1500,1985$.

5. V. Yu. Kistenev, L. S. Kuzmin, A. G. Odintsov, E. A. Polunin, and L. Yu. Syromyatnikov, "Properties of the lead-alloy Josephson tunnel junctions" pp. 113-118 in SQUID-85, H. D. Hahlbohm and H. Lübbig, Eds., W. de Gruyer \& Co., Berlin, 1985 .

6. L. Kuzmin, H. K. Olsson, and T. Claeson, "Parametric effects in inductively compensated tunnel junctions", pp. 1017-1022 in ibid.

7. H. D. Jensen, A. Larsen, and J. Mygind, "Parametrically generated steps and chaos in a Josephson junction coupled to a resonator", submitted to J. Appl. Phys.

8. A. Larsen, H. D. Jensen, and J. Mygind, "Self-induced steps in a small Josephson junction strongly coupled to a multi-mode resonator", submitted to Phys. Rev. B. 